\title{
ARTICLE
}

Lymphoma

\section{Single-agent activity of phosphatidylinositol 3-kinase inhibition with copanlisib in patients with molecularly defined relapsed or refractory diffuse large B-cell lymphoma}

\author{
Georg Lenz ${ }^{1} \cdot$ Eliza Hawkes $^{2} \cdot$ Gregor Verhoef $^{3} \cdot$ Corinne Haioun $^{4}$ Soon Thye Lim $^{5} \cdot$ Dae Seog Heo $^{6}$.

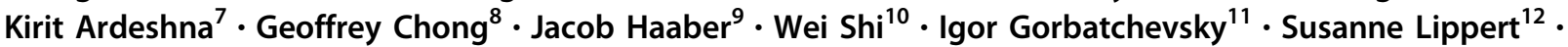 \\ Florian Hiemeyer $^{12}$ - Paolo Piraino ${ }^{12} \cdot$ Georg Beckmann $^{12} \cdot$ Carol Peña $^{11} \cdot$ Viktoriya Buvaylo $^{11} \cdot$ Barrett H. Childs $^{11}$. \\ Gilles Salles ${ }^{13}$
}

Received: 21 November 2019 / Revised: 30 January 2020 / Accepted: 3 February 2020 / Published online: 14 February 2020

(c) The Author(s) 2020. This article is published with open access

\begin{abstract}
Patients with relapsed/refractory diffuse large B-cell lymphoma (DLBCL) have adverse outcomes. We evaluated the efficacy and safety of the phosphatidylinositol 3-kinase inhibitor copanlisib in patients with relapsed/refractory DLBCL and assessed the relationship between efficacy and DLBCL cell of origin (COO; activated B-cell like [ABC] and germinal center B-cell like $[\mathrm{GCB}])$ and other biomarkers. The primary endpoint was objective response rate (ORR) in DLBCL COO subgroups (ABC, GCB, and unclassifiable) and by $C D 79 B$ mutational status (NCT02391116). Sixty-seven patients received copanlisib (ABC DLBCL, $n=19$; GCB DLBCL, $n=30$; unclassifiable, $n=3$; missing, $n=15$ ). The ORR was $19.4 \%$; $31.6 \%$ and $13.3 \%$ in ABC and GCB DLBCL patients, respectively. ORR was $22.2 \% / 20.0 \%$ for patients with/without $C D 79 B$ mutations (wild type, $n=45$; mutant, $n=9$; missing, $n=13$ ). Overall median progression-free survival and duration of response were 1.8 and 4.3 months, respectively. Adverse events included hypertension (40.3\%), diarrhea (37.3\%), and hyperglycemia (32.8\%). Aberrations were detected in 338 genes, including BCL2 (53.7\%) and MLL2 (53.7\%). A 16-gene signature separating responders from nonresponders was identified. Copanlisib treatment demonstrated a manageable safety profile in patients with relapsed/refractory DLBCL and a numerically higher response rate in ABC vs. GCB DLBCL patients.
\end{abstract}

Supplementary information The online version of this article (https:// doi.org/10.1038/s41375-020-0743-y) contains supplementary material, which is available to authorized users.

Georg Lenz

georg.lenz@ukmuenster.de

1 Department of Medicine A, Hematology, Oncology, and Pneumology, University Hospital Münster, Münster, Germany

2 Eastern Health Clinical School, Monash University, Olivia Newton John Cancer Research and Wellness Centre, Melbourne, VIC, Australia

3 University Hospitals Leuven, Leuven, Belgium

4 Lymphoid Malignancies Unit, Groupe Hospitalier Henri MondorAlbert Chenevier, Creteil, France

5 National Cancer Centre Singapore and Duke-NUS Medical School, Singapore, Singapore

6 Department of Internal Medicine, Seoul National University

\section{Introduction}

Malignant lymphoma encompasses a heterogeneous group of malignancies [1]. Diffuse large B-cell lymphoma (DLBCL), characterized by aggressive clinical behavior, is

Hospital, Seoul, South Korea

7 University College London Hospitals NHS Foundation Trust, London, UK

8 Ballarat Regional Integrated Cancer Centre, Ballarat, VIC, Australia

9 Department of Hematology, Odense University Hospital, Odense, Denmark

10 Bayer China, Beijing, China

11 Bayer HealthCare Pharmaceuticals, Inc., Whippany, NJ, USA

12 Pharmaceuticals Division, Bayer AG, Berlin, Germany

13 Hospices Civils de Lyon, Université de Lyon, Centre Hospitalier Lyon-Sud, Service d'hématologie, Lyon, France 
the most frequent subtype, accounting for $\sim 30-40 \%$ of new cases diagnosed globally [2].

While most patients with DLBCL achieve a durable response to standard-of-care first-line chemotherapy of rituximab with cyclophosphamide, doxorubicin, vincristine, and prednisone (CHOP) [3, 4], approximately one-third of patients develop disease that is refractory to, or has relapsed after, initial response [5]. Patients with relapsed/refractory DLBCL are characterized by adverse prognosis $[6,7]$. High-dose chemotherapy followed by autologous stem cell transplantation remains the current standard of care for patients with relapsed/refractory DLBCL, although longterm outcomes are poor [7]. In addition, some patients are ineligible due to age or contraindicating comorbidities $[5,8]$. Novel therapies are urgently required to improve outcomes for DLBCL patients.

Gene-expression profiling distinguishes at least two major molecular DLBCL subtypes, reflecting the cell of origin (COO): activated B-cell-like (ABC) DLBCL and germinal center B-cell-like (GCB) DLBCL [1, 9]. These subtypes differ in their gene-expression profiles, clinical outcomes, prognosis, and responsiveness to targeted therapies [10, 11]. $A B C$ DLBCLs frequently harbor $C D 79 B$ mutations, resulting in chronic active B-cell receptor (BCR) signaling, an important pathogenetic mechanism in these lymphomas [12]. In preclinical studies, targeted inhibition of the downstream BCRsignaling molecules phosphatidylinositol 3-kinase (PI3K)- $\alpha$ and $-\delta$ has demonstrated significant antilymphoma activity, particularly in models of ABC DLBCL [13-15]. In addition, in vitro and in vivo data have suggested that $A B C$ DLBCL models bearing $C D 79 B$ and/or MYD88 mutations may be more sensitive to inhibition of PI3K- $\alpha$ and $-\delta$ isoforms than other molecular DLBCL subtypes [14]. Whereas data from a small phase I study indicated that selective inhibition of PI3K$\delta$ was ineffective in treating DLBCL [16], subsequent preclinical studies demonstrated that dual inhibition of PI3K- $\alpha$ and $-\delta$ isoforms may be necessary for effective treatment of DLBCL $[14,15,17]$. Indeed, inhibition of PI3K- $\delta$ alone may be self-limiting as it has been shown to result in feedback activation of PI3K- $\alpha$ and BCR signaling in ABC DLBCL cell lines [17].

Copanlisib (Aliqopa; Bayer AG, Berlin, Germany) is a pan-class I PI3K inhibitor with potent activity against PI3K$\alpha$ and $-\delta$ isoforms [18]. A first-in-human phase I study determined the maximum tolerated dose of copanlisib to be $0.8 \mathrm{mg} / \mathrm{kg}$ administered on days 1,8 , and 15 of a 28 -day cycle, with an expansion cohort demonstrating satisfactory safety and a partial response (PR) in a patient with unselected DLBCL (objective response rate [ORR] 1/3 patients; $33 \%$ ) on treatment more than 16 months [19]. A phase II study in 48 patients with aggressive lymphoma, including 15 patients with unselected DLBCL, observed an overall ORR of $27 \%$ with one PR in a DLBCL patient [20]. Lastly, a recent phase II study of copanlisib in pretreated patients with relapsed/refractory indolent lymphoma demonstrated promising efficacy (ORR 84/142 patients; 59\%) and manageable toxicity [21]. Based on these results, we conducted a phase II study evaluating the efficacy and safety of copanlisib in patients with relapsed/refractory DLBCL. We also evaluated the molecular profiles of patients to potentially identify subgroups that may benefit most from copanlisib by assessing the relationships between efficacy and these potential candidate predictive biomarkers. We believe this is the largest study to date investigating a PI3K inhibitor in patients with relapsed or refractory DLBCL.

\section{Materials and methods}

\section{Study design and participants}

This single-arm, open-label, multicenter, phase II study (NCT02391116) evaluated the efficacy and safety of singleagent copanlisib in patients with relapsed/refractory DLBCL. Patients eligible for inclusion were aged $\geq 18$ years, with a histologically confirmed diagnosis of de novo DLBCL, or DLBCL transformed from follicular lymphoma (FL) according to the World Health Organization classification [22]. Eligible patients had received one or more lines of prior therapy for DLBCL, including rituximab with $\mathrm{CHOP}$ or CHOP-like regimens, and were not eligible or not willing to receive high-dose myeloablative chemotherapy and stem cell transplantation. Additional eligibility and exclusion criteria are included in the Supplementary information.

The study was compliant with the Declaration of Helsinki and Good Clinical Practice and was approved by the appropriate ethics committees prior to the start of recruitment. All participants provided written, informed consent before study entry.

\section{Copanlisib treatment}

Patients received copanlisib $60 \mathrm{mg}$ as a $1 \mathrm{~h}$ intravenous infusion on days 1,8 , and 15 of a 28-day cycle. Dose reductions to $45 \mathrm{mg}$ were permitted in the event of toxicities. Treatment continued until disease progression or unacceptable toxicity. All patients completed a 30-day safety follow-up after the last dose except for patients lost to follow-up or who had died. Patients who discontinued treatment for any reason other than radiologic disease progression entered active follow-up, which encompassed the safety follow-up.

\section{Objectives and assessments}

The primary objective was to assess the efficacy of copanlisib in patients with relapsed/refractory DLBCL and 
to evaluate the relationship between efficacy and potentially predictive biomarkers, specifically DLBCL COO and $C D 79 B$ mutational status. Secondary objectives were to assess other radiologic and survival indicators of treatment efficacy, safety, and tolerability of copanlisib, and long-term effects of treatment.

The primary efficacy variable was ORR, defined as the proportion of patients who had an overall response of complete response (CR) or PR according to Lugano 2014 criteria [23], based on the investigator's assessment; these patients were considered to have shown an objective response and were deemed responders. Response was assessed in biomarker subgroups based on DLBCL COO (ABC, GCB, or unclassifiable) and $C D 79 B$ mutational status. All other biomarker analyses were exploratory.

Secondary efficacy variables included duration of response (DoR), progression-free survival (PFS), overall survival, duration of stable disease, and disease control rate (DCR); these variables are defined in the Supplementary information.

Radiologic tumor assessments by computed tomography/ magnetic resonance imaging scan or positron emission tomography-computed tomography scan were performed at baseline, every 8 weeks during treatment, and every 12 weeks during active follow-up. Safety was assessed continually throughout the study through clinical laboratory variables, physical examinations, Eastern Cooperative Oncology Group performance status, vital signs, 12-lead electrocardiogram, and cardiac function. Adverse events (AEs) were graded using the National Cancer Institute Common Terminology Criteria for Adverse Events version 4.03.

The overall cohort was defined as patients assigned to treatment (full analysis set). The per-protocol set (PPS) was defined as patients from the overall cohort who had a tumor biopsy available at baseline ( $\mathrm{COO}$ and $C D 79 B$ results), completed at least one treatment cycle (three doses), had no major protocol deviation affecting the primary efficacy evaluation, and had at least one postbaseline tumor assessment (or discontinuation due to death or progression by clinical judgement before tumor assessment). Results of the primary analysis are presented here for the PPS and overall cohort. Safety variables were analyzed using the overall cohort. Additional results are presented for the overall cohort, with additional results for the PPS available in the Supplementary information.

\section{CD79B and DLBCL COO assays and biomarker analysis}

Pretreatment tumor biopsy samples were mandatory for biomarker analysis (either fresh samples collected at screening and/or archival formalin-fixed, paraffin- embedded samples collected after the last relapse or disease progression). $C D 79 B$ mutational status and other variants were assayed using next-generation sequencing (NGS) with the FoundationOne Heme panel (Foundation Medicine, Cambridge, MA, USA), which tested for variants in $\geq 400$ genes, including selected rearrangements/fusions frequently reported in hematological malignancies. DLBCL $\mathrm{COO}$ was determined using the EdgeSeq $\mathrm{COO}$ assay (HTG Molecular Diagnostics, Inc., Tucson, AZ, USA [HTG EDGE-SQ-100]) based on mRNA-expression levels of a 22-gene panel.

\section{Statistical methods}

The primary efficacy analysis of ORR was performed on the overall cohort and PPS for DLBCL COO and CD79B biomarkers, with PPS being the primary analysis set as it allowed for precise estimation and comparison of the $\mathrm{COO}$ and $C D 79 B$ biomarker effect. Differences of ORR in the respective biomarker-positive group minus ORR in the complementary (biomarker-negative) group were calculated. Twosided $90 \%$ confidence intervals (CIs) for the differences were provided. $P$ values for exploratory purpose were calculated using Barnard's unconditional exact test. ORRs for the PPS and overall cohort were also calculated. For the ORR, exact two-sided 90\% CIs based on Clopper-Pearson methodology were provided. For secondary efficacy variables, the overall cohort was used for the main analysis. DoR, PFS, overall survival, and duration of stable disease were presented using the Kaplan-Meier method, and medians with $95 \%$ CIs were calculated. The study was conceptualized as an estimation study and sample size was chosen based on expected length of the CI for ORR difference. Additional methods for sample size planning and statistical power can be found in the Supplementary information.

Additional mutational status (excluding those for the primary efficacy analysis) was evaluated for correlation with clinical endpoints, using Fisher's exact test for response and disease control endpoints, and Kaplan-Meier plots as well as hazard ratios determined by Cox regression models for PFS.

Exploratory analysis of mutation-based signatures was performed. A subset of 134 genes was analyzed based on the presence of at least three patients with mutations in these genes. Correspondence discriminant analysis [24] was used to separate samples on the basis of investigator-assessed treatment response, and a composite score was calculated; additional details are provided in the Supplementary information.

\section{Results}

Sixty-seven patients were assigned to treatment and comprised the overall cohort (Table 1). The median age at 
Table 1 Patient demographics and baseline characteristics by ABC DLBCL, GCB DLBCL, and in the overall cohort.

\begin{tabular}{|c|c|c|c|}
\hline & $\begin{array}{l}\text { ABC DLBCL } \\
n=19\end{array}$ & $\begin{array}{l}\text { GCB DLBCL } \\
n=30\end{array}$ & $\begin{array}{l}\text { Overall cohort } \\
N=67^{\mathrm{a}}\end{array}$ \\
\hline Age (years), median (range) & $72.0(41-84)$ & $67.0(32-85)$ & $69.0(25-93)$ \\
\hline Age $\geq 65$ years, $n(\%)$ & $15(78.9)$ & $17(56.7)$ & $40(59.7)$ \\
\hline Male, $n(\%)$ & $11(57.9)$ & $17(56.7)$ & $39(58.2)$ \\
\hline \multicolumn{4}{|l|}{ ECOG performance status score, $n(\%)$} \\
\hline 0 & $5(26.3)$ & $8(26.7)$ & $15(22.4)$ \\
\hline 1 & $11(57.9)$ & $16(53.3)$ & $38(56.7)$ \\
\hline 2 & $3(15.8)$ & $6(20.0)$ & $14(20.9)$ \\
\hline \multicolumn{4}{|l|}{ Histology of tumor, $n(\%)$} \\
\hline DLBCL transformed from FL & $3(15.8)$ & $9(30.0)$ & $14(20.9)$ \\
\hline DLBCL not otherwise specified & $16(84.2)$ & $21(70.0)$ & $51(76.1)$ \\
\hline EBV-positive DLBCL of the elderly & 0 & 0 & $1(1.5)$ \\
\hline T-cell/histocyte-rich large B-cell lymphoma & 0 & 0 & $1(1.5)$ \\
\hline \multicolumn{4}{|l|}{ Stage at study entry, $n(\%)$} \\
\hline I & 0 & $3(10.0)$ & $3(4.5)$ \\
\hline II & 0 & $6(20.0)$ & $9(13.4)$ \\
\hline III & $4(21.1)$ & $7(23.3)$ & $11(16.4)$ \\
\hline IV & $15(78.9)$ & $14(46.7)$ & $44(65.7)$ \\
\hline $\begin{array}{l}\text { Median time from initial diagnosis to start of study } \\
\text { treatment, months (range) }\end{array}$ & $16.8(1.4-123)$ & $41.4(0.7-192)$ & $17.1(0.7-192)$ \\
\hline Median time since first progression, months (range) & $3.0(0.2-60.5)$ & $6.9(0.7-184)$ & $4.3(0.2-184)$ \\
\hline $\begin{array}{l}\text { Median time from most recent progression to start of } \\
\text { study treatment, months (range) }\end{array}$ & $0.8(0.2-3.0)$ & $1.3(0.2-6.2)$ & $5.0(0.2-6.2)$ \\
\hline Median prior anticancer therapy lines, $n$ (range) & $2(1-5)$ & $3.5(1-13)$ & $3(1-13)$ \\
\hline $\begin{array}{l}\text { Median time since last systemic anticancer therapy, } \\
\text { months (range) }\end{array}$ & $1.7(0.7-118)$ & $1.6(1.0-68.5)$ & $1.9(0.7-118)$ \\
\hline \multicolumn{4}{|l|}{ Refractory against last systemic anticancer therapy, $n(\%)$} \\
\hline Yes & $15(78.9)$ & $24(80.0)$ & $52(77.6)$ \\
\hline No & $4(21.1)$ & $6(20.0)$ & $15(22.4)$ \\
\hline
\end{tabular}

$A B C$ activated B-cell like, $C O O$ cell of origin, $D L B C L$ diffuse large B-cell lymphoma, $E B V$ Epstein-Barr virus, ECOG Eastern Cooperative Oncology Group, $F L$ follicular lymphoma, $G C B$ germinal center B-cell like.

ancludes three unclassifiable patients and 15 patients with data missing for COO.

baseline was 69 years (range 25-93), 58.2\% were male, and the majority of patients $(56.7 \%)$ had an Eastern Cooperative Oncology Group performance status of 1. Patients had received a median of three (range 1-13) prior lines of antilymphoma therapy, and 52 patients $(77.6 \%)$ were refractory against their last systemic treatment (Table 1). Two patients (3.0\%), one each with ABC DLBCL and GCB DLBCL, had received a prior autologous stem cell transplant. Fourteen patients had DLBCL transformed from FL. Fifty-six patients entered the safety follow-up.

Nineteen patients had ABC DLBCL, 30 patients had GCB DLBCL, three patients were unclassifiable, and 15 patients did not have a valid assessment and were deemed missing due to lack of adequate patient material. For those patients evaluable for DLBCL COO, patients with $\mathrm{ABC}$ DLBCL had a numerically lower incidence of DLBCL transformed from FL ( $15.8 \%$ vs. $30.0 \%)$, a higher incidence of stage IV disease (78.9\% vs. $46.7 \%$ ), a shorter median time from diagnosis to start of copanlisib treatment (16.8 months vs. 41.4 months), and had received numerically fewer prior lines of therapy (2 vs. 3.5 ) compared with patients with GCB DLBCL (Table 1). A similar proportion of patients with ABC DLBCL and GCB DLBCL were refractory against the last systemic therapy $(78.9 \%$ vs. $80.0 \%$ ) (Fig. 1).

NGS data were available for 54 patients, 45 of whom had wild-type $C D 79 B$ and nine of whom had mutant $C D 79 B$; NGS data were missing for 13 patients due to lack of adequate material.

Forty patients comprised the PPS (Supplementary Table 1). The main reason for exclusion (18/27 patients) was lack of baseline $\mathrm{COO}$ assessment and/or $C D 79 B$ 
A

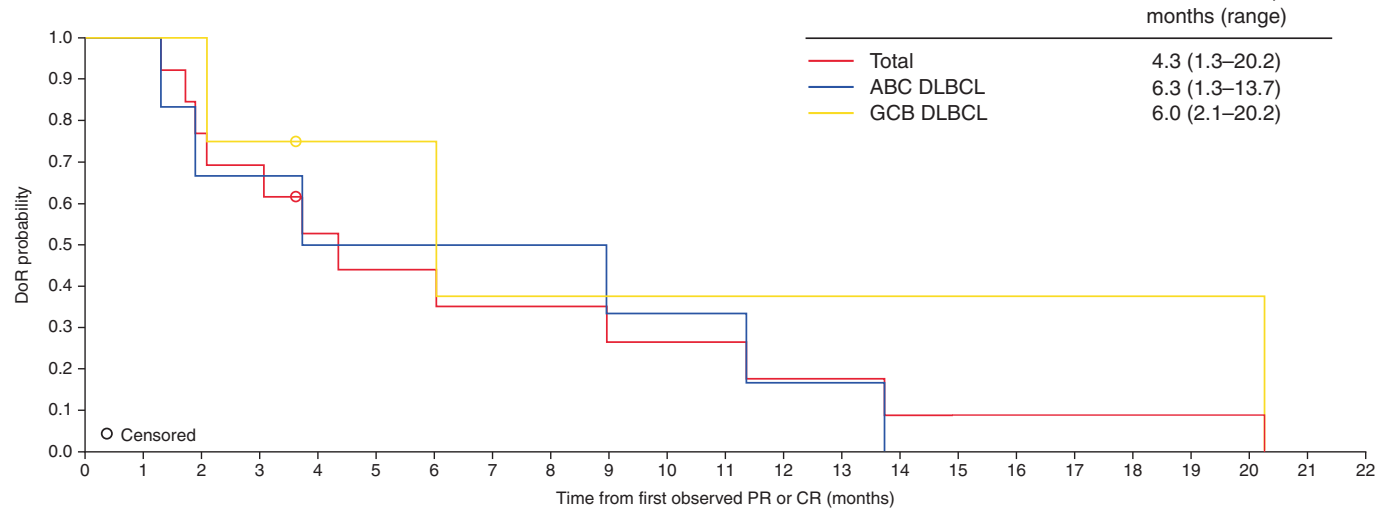

Number of patients at risk

\begin{tabular}{rcccccccccccccc}
\hline Total & 13 & 12 & 8 & 5 & 4 & 4 & 3 & 2 & 2 & 1 & 1 & 1 & 1 & 0 \\
ABC DLBCL & 6 & 5 & 4 & 3 & 3 & 3 & 2 & 1 & 1 & 0 & 0 & 0 & 0 & 0 \\
GCB DLBCL & 4 & 4 & 3 & 2 & 1 & 1 & 1 & 1 & 1 & 1 & 1 & 1 & 1 & 0
\end{tabular}

B

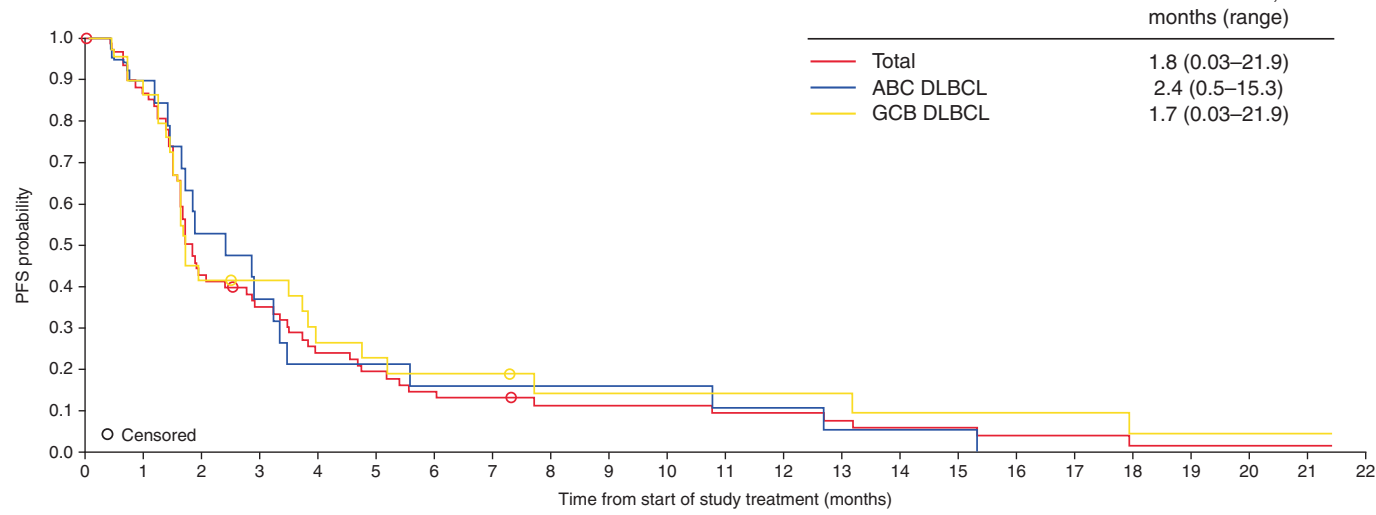

Number of patients at risk

\begin{tabular}{|c|c|c|c|c|c|c|c|c|c|c|c|c|c|c|}
\hline Total & 67 & 43 & 21 & 12 & 8 & 6 & 6 & 5 & 4 & 3 & 2 & 1 & 1 & 1 \\
\hline ABC DLBCL & 19 & 14 & 6 & 4 & 3 & 3 & 3 & 2 & 1 & 1 & 0 & 0 & 0 & 0 \\
\hline
\end{tabular}

\section{C}

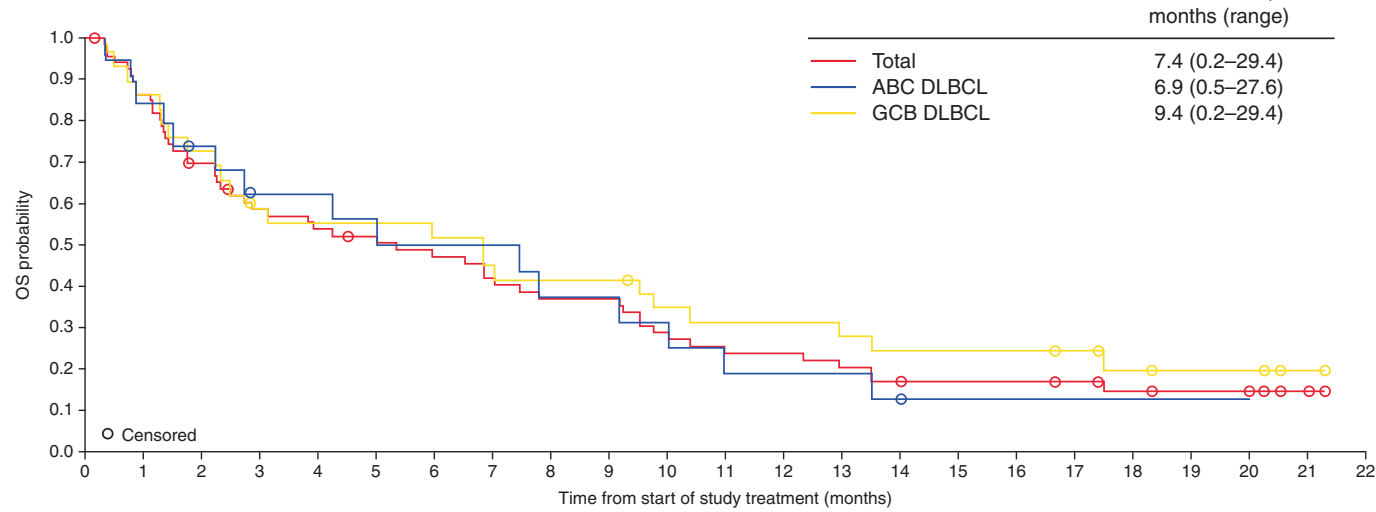

\begin{tabular}{c} 
Number of patients at risk \\
\hline Total
\end{tabular}

Fig. 1 Kaplan-Meier curves. a Median overall DoR; b Median PFS; and c Median OS (overall cohort). ABC activated B-cell like, DLBCL diffuse large B-cell lymphoma, DoR duration of response, GCB germinal center B-cell like, PFS progression-free survival, OS overall survival. 
Table 2 Tumor response based on investigator assessment. a Tumor response in the overall cohort; $\mathbf{b}$ objective response rate in molecular DLBCL subtypes in the overall cohort and PPS.

(A) Tumor response in the overall cohort

\begin{tabular}{|c|c|c|c|c|c|c|c|c|}
\hline & \multirow[t]{2}{*}{$\begin{array}{l}\text { Total } \\
N=67\end{array}$} & \multicolumn{3}{|c|}{$\begin{array}{l}C D 79 B \text { mutational status } \\
n=67\end{array}$} & \multicolumn{4}{|c|}{$\begin{array}{l}\text { DLBCL COO subgroup } \\
n=67\end{array}$} \\
\hline & & $\begin{array}{l}\text { Mutant } \\
C D 79 B \\
n=9\end{array}$ & $\begin{array}{l}\text { Wild-type } \\
C D 79 B \\
n=45\end{array}$ & $\begin{array}{l}\text { Missing } \\
n=13\end{array}$ & $\begin{array}{l}\text { ABC DLBCL } \\
n=19\end{array}$ & $\begin{array}{l}\text { GCB DLBCL } \\
n=30\end{array}$ & $\begin{array}{l}\text { Unclassifiable } \\
n=3\end{array}$ & $\begin{array}{l}\text { Missing } \\
n=15\end{array}$ \\
\hline \multicolumn{9}{|c|}{ Best overall response, $n(\%)$} \\
\hline $\mathrm{CR}$ & $5(7.5)$ & $1(11.1)$ & $4(8.9)$ & 0 & $4(21.1)$ & $1(3.3)$ & 0 & 0 \\
\hline PR & $8(11.9)$ & $1(11.1)$ & $5(11.1)$ & $2(15.4)$ & $2(10.5)$ & $3(10.0)$ & $1(33.3)$ & $2(13.3)$ \\
\hline Stable disease & $14(20.9)$ & $3(33.3)$ & $9(20.0)$ & $2(15.4)$ & $4(21.1)$ & $8(26.7)$ & 0 & $2(13.3)$ \\
\hline Progressive disease & $30(44.8)$ & $4(44.4)$ & $17(37.8)$ & $9(69.2)$ & $7(36.8)$ & $13(43.3)$ & $2(66.7)$ & $8(53.3)$ \\
\hline $\begin{array}{l}\text { Not evaluable/not } \\
\text { available }^{\mathrm{a}}\end{array}$ & $10(14.9)$ & 0 & $10(22.2)$ & 0 & $2(10.5)$ & $5(16.7)$ & 0 & $3(20.0)$ \\
\hline ORR, $n(\%)$ & $13(19.4)$ & $2(22.2)$ & $9(20.0)$ & $2(15.4)$ & $6(31.6)$ & $4(13.3)$ & $1(33.3)$ & $2(13.3)$ \\
\hline $90 \% \mathrm{CI}$ & $11.9,29.1$ & $4.1,55.0$ & $10.9,32.3$ & $2.8,41.0$ & $14.7,53.0$ & $4.7,28.0$ & $1.7,86.5$ & $2.4,36.3$ \\
\hline DCR, $n(\%)$ & $27(40.3)$ & $5(55.6)$ & $18(40.0)$ & $4(30.8)$ & $10(52.6)$ & $12(40.0)$ & $1(33.3)$ & $4(26.7)$ \\
\hline
\end{tabular}

(B) Objective response rate in molecular DLBCL subtypes in the overall cohort and PPS

\begin{tabular}{|c|c|c|c|c|c|c|}
\hline \multirow[t]{2}{*}{ Biomarker } & \multicolumn{3}{|l|}{ Overall cohort } & \multicolumn{3}{|l|}{ PPS } \\
\hline & ORR, \% & ORR difference, $\%(90 \% \mathrm{CI})$ & $P$ value & ORR, \% & ORR difference, $\%(90 \% \mathrm{CI})$ & $P$ value \\
\hline $\mathrm{ABC}$ vs. GCB & 31.6 vs. 13.3 & $18.2(-6.1,41.0)$ & 0.1413 & 37.5 vs. 13.6 & $23.9(-3.3,48.3)$ & 0.1074 \\
\hline $\mathrm{ABC}$ vs. GCB plus unclassifiable & 31.6 vs. 15.2 & $16.4(-7.2,39.1)$ & 0.1751 & 37.5 vs. 16.7 & $20.8(-6.8,46.2)$ & 0.1638 \\
\hline GCB vs. ABC plus unclassifiable & 13.3 vs. 31.8 & $-18.5(-40.1,4.6)$ & 0.1329 & 13.6 vs. 38.9 & $-25.3(-49.1,1.1)$ & 0.0762 \\
\hline Unclassifiable $^{\mathrm{b}}$ & 33.3 & & & 50.0 & & \\
\hline
\end{tabular}

$A B C$ activated B-cell like, $C I$ confidence interval, $C O O$ cell of origin, $C R$ complete response, $D L B C L$ diffuse large B-cell lymphoma, $G C B$ germinal center B-cell like, $O R R$ objective response rate, $P P S$ per-protocol set, $P R$ partial response.

${ }^{a}$ One patient had postbaseline tumor assessment(s) that could not be evaluated by the investigator and nine patients had no postbaseline tumor assessment due to discontinuation because of progression by clinical judgement, death occurring before disease was reevaluated, or other reasons; these ten patients were considered nonresponders.

${ }^{\mathrm{b}}$ Overall cohort, $n=3$; PPS, $n=2$; no statistical comparisons reported due to small sample size.

mutational assessment due to lack of adequate material. Of the 40 patients in the PPS, 16 had ABC DLBCL, six of whom had mutant $C D 79 B$. In addition, 22 patients had GCB DLBCL, two of whom had mutant CD79B. Two patients had an unclassifiable DLBCL subtype. Ten patients had DLBCL transformed from FL.

Median overall extent of exposure was 1.6 months (range $0.2-16.6$ ), corresponding to a median of 1.75 cycles (range 0.3-18.0). Patients received a median of six infusions (range 1-54). At the database cutoff, no patients were ongoing with study treatment.

In the overall cohort, the ORR was $19.4 \%$ : five patients (7.5\%) achieved CR and eight patients (11.9\%) achieved PR. The DCR was $40.3 \%$ (Table 2a). The ORR was $31.6 \%$ (6/19 patients) for ABC DLBCL patients and 13.3\% (4/30 patients) for GCB DLBCL patients $(P=0.1413)$ or, similarly, $15.2 \%$ (5/33 patients) for GCB DLBCL plus unclassifiable patients $(P=0.1751)$. Accordingly, GCB DLBCL patients had an $18.5 \%$ lower ORR than ABC DLBCL plus unclassifiable patients $(P=0.1329)$ (Table $2 \mathrm{~b})$. Median DoR was 4.3 months (range 1.3-20.2) (6.3 [range 1.3-13.7] and 6.0 months [range 2.1-20.2] for patients with $\mathrm{ABC}$ DLBCL and GCB DLBCL, respectively) (Fig. 1a). Median duration of stable disease was 3.5 months (range 1.5-17.9). Of the 39 patients with calculable percentage changes from baseline in target lesions, $46.7 \%$ (7/15) and $16.7 \%$ (4/24) of ABC DLBCL and GCB DLBCL patients, respectively, had a $\geq 50 \%$ reduction in lesion size (Fig. 2).

For patients with mutant $C D 79 B$, the ORR was $22.2 \%$ (2/9 patients); for those with wild-type $C D 79 B$, ORR was $20.0 \%$ (9/45 patients) (Table 2a). Of the patients who had DLBCL transformed from FL, ORR was $21.4 \%$ (3/14 patients: one $\mathrm{CR}$; two PR).

Analysis of ORR in the PPS subgroup of patients with more complete molecular profiling information available demonstrated similar results to the overall cohort (Table $2 b$, 
Fig. 2 Waterfall plot of percentage best change in (investigator assessment) in patients with ABC DLBCL and GCB DLBCL (overall cohort). ABC activated B-cell like, DLBCL diffuse large B-cell lymphoma, GCB germinal center B-cell like. target lesion size from baseline

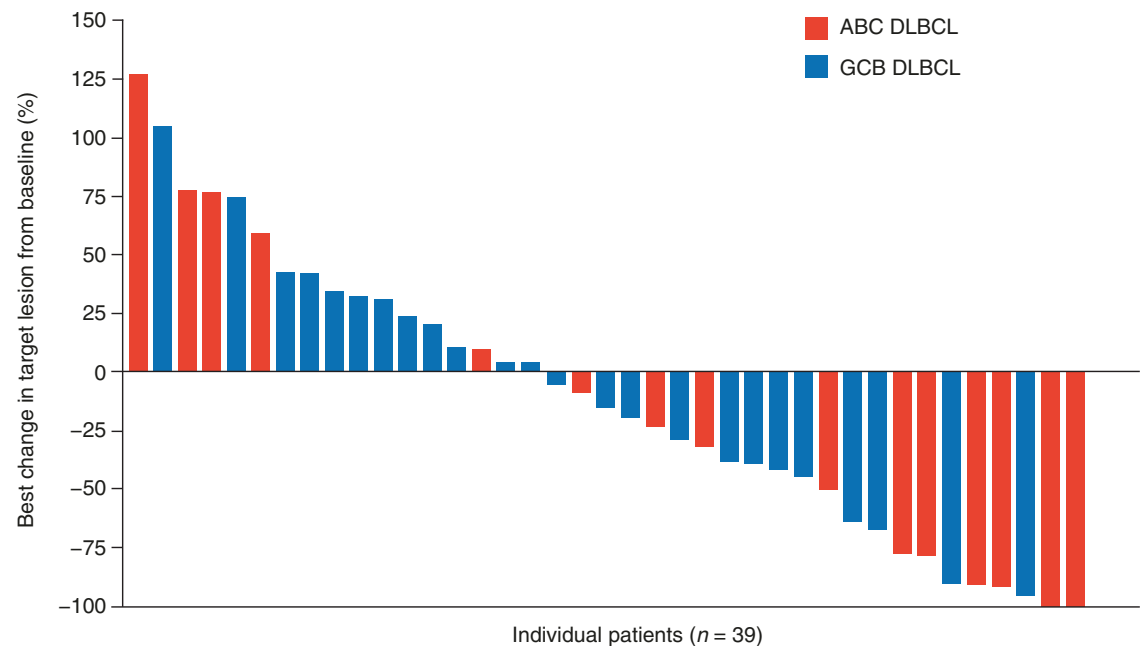

Supplementary Table 2). Comparing pairwise differences for ORR in the PPS subgroups, ABC DLBCL patients had a response rate of $37.5 \%$, whereas GCB DLBCL patients had a response rate of $13.6 \%$. ABC DLBCL patients had a 20.8\% higher ORR than GCB DLBCL plus unclassifiable patients $(P=0.1638)$, and GCB DLBCL patients had an ORR 25.3\% lower than ABC DLBCL plus unclassifiable patients $(P=0.0762)$. Median DoR in the PPS subgroup was 3.7 months (range 1.3-20.2) (6.3 [range 1.3-13.7] and 20.2 months [range 2.1-20.2] for patients with $\mathrm{ABC}$ DLBCL and GCB DLBCL, respectively) (Supplementary Fig. 1a).

The median overall PFS in the overall cohort was 1.8 months (range 0.03-21.9). Estimated PFS rates at 3, 6, 9 , and 12 months were $34.7 \%, 14.2 \%, 10.8 \%$, and $9.0 \%$, respectively. Median PFS was 2.4 months (range 0.5-15.3) for ABC DLBCL patients and 1.7 months (range 0.03-21.9) for GCB DLBCL patients (Fig. 1b). Median OS was 7.4 months (range 0.2-29.4) (Fig. 1c), and estimated OS rates at 3,6, 9, and 12 months were $69.7 \%, 52.2 \%, 45.4 \%$, and $37.0 \%$, respectively.

Median overall PFS in the PPS subgroup was 2.8 months (range 0.7-21.9), with estimated PFS rates of 44.6\%, $18.4 \%$, and $15.3 \%$ at 3,6 , and 12 months, respectively (Supplementary Fig. 1b). Median PFS was 2.9 months (range 0.7-15.3) for ABC DLBCL patients and 1.8 months (range 1.2-21.9) for GCB DLBCL patients.

Of the 54 patient samples with targeted NGS data available, 338 genes with aberrations were detected. Samples had a median of 24.5 (range 5-61) alterations detected by NGS. Overall, the most common alterations included BCL2 (29 patient samples, 53.7\%; predominantly gene rearrangements), MLL2 (29 patient samples, 53.7\%), TP53 (22 patient samples, 40.7\%), BCL6 (16 patient samples, $29.6 \%$; mainly gene rearrangements with a few short variants), and MYC (12 patient samples, 22.2\%; mostly rearrangements, some short variants and copy-number alterations) (Fig. 3a), with differences observed between the COO groups (Fig. 3b, c, Supplementary Table 3, Supplementary Fig. 2).

Forty-four patients had both NGS biomarker data and investigator-assessed response data available (Fig. 4a, Supplementary Table 4). Of genes known to be associated with constitutive oncogenic nuclear factor- $\mathrm{\kappa B}$ signaling, the ORR for patients with and without MYD88 mutations was $25.0 \%$ ( $2 / 8$ patients and 9/36 patients, respectively), while patients with mutated TNFAIP3 demonstrated a better ORR than those with wild-type $(57.1 \%$ [ $4 / 7$ patients] vs. $18.9 \%$ [7/37 patients]; $P=0.054$ ). Patients with NFKBIA mutations had worse outcomes than those with wild-type NFKBIA (PFS: hazard ratio 0.21, $P=0.013$; DCR: $0 \%$ [0/ 3 patients] vs. $56.1 \%$ [23/41 patients], $P=0.100$ ). $C A R D 11$ wild-type and CARDI1-mutant patients had ORRs of $27.0 \%$ (10/37 patients) and $14.3 \%$ (1/7 patients), respectively, and DCRs of $56.8 \%$ (21/37 patients) and 28.6\% (2/7 patients), respectively.

Response rates for patients with and without $B C L 2$ were $16.7 \%$ (4/24 patients) and $35.0 \%$ (7/20 patients), respectively. Of the six patients with alterations in both $M Y C$ and either $B C L 2$ or $B C L 6$, no responders were identified (Fig. 4a, Supplementary Table 4), compared with an ORR of $28.9 \%$ (11/38 patients) in patients without both alterations. While the group sizes in most of these exploratory analyses are small, the findings suggest hypotheses that can be investigated in future studies.

To better understand these observed responses to copanlisib, we performed an unbiased exploratory analysis that identified a 16-gene mutation signature separating responders from nonresponders (Fig. 4b). A composite score was calculated, reflecting numerically the collective presence of mutations in specific genes in our resulting gene set, along with the absence of mutations in others. Patients with a high composite score had a higher ORR and longer PFS compared with those with a low composite score (ORR 
A

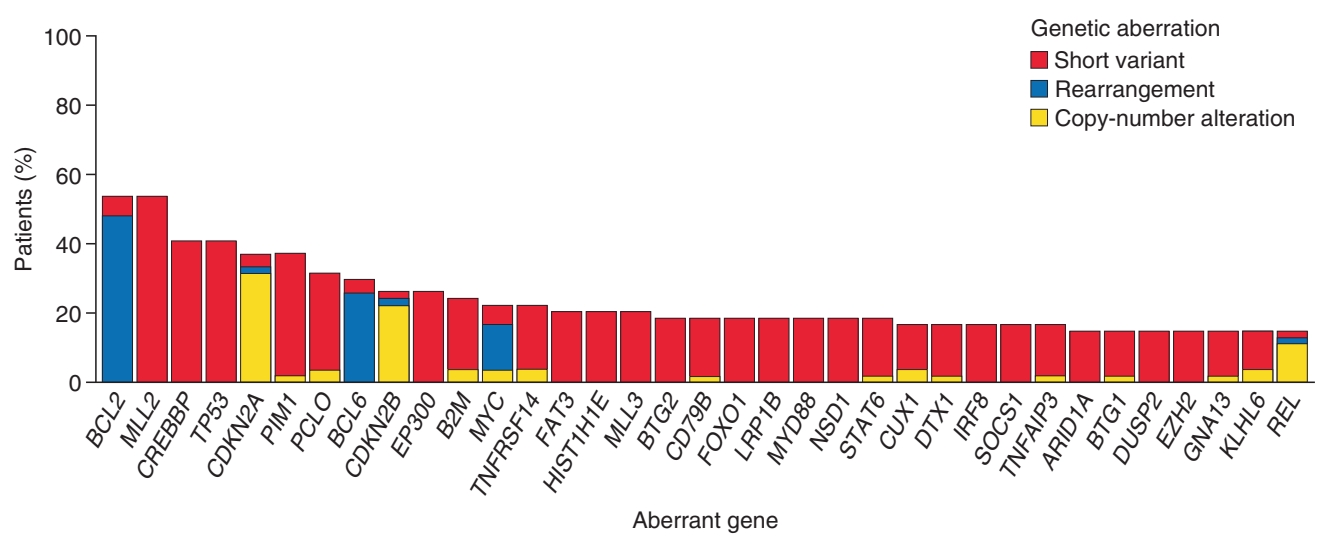

B

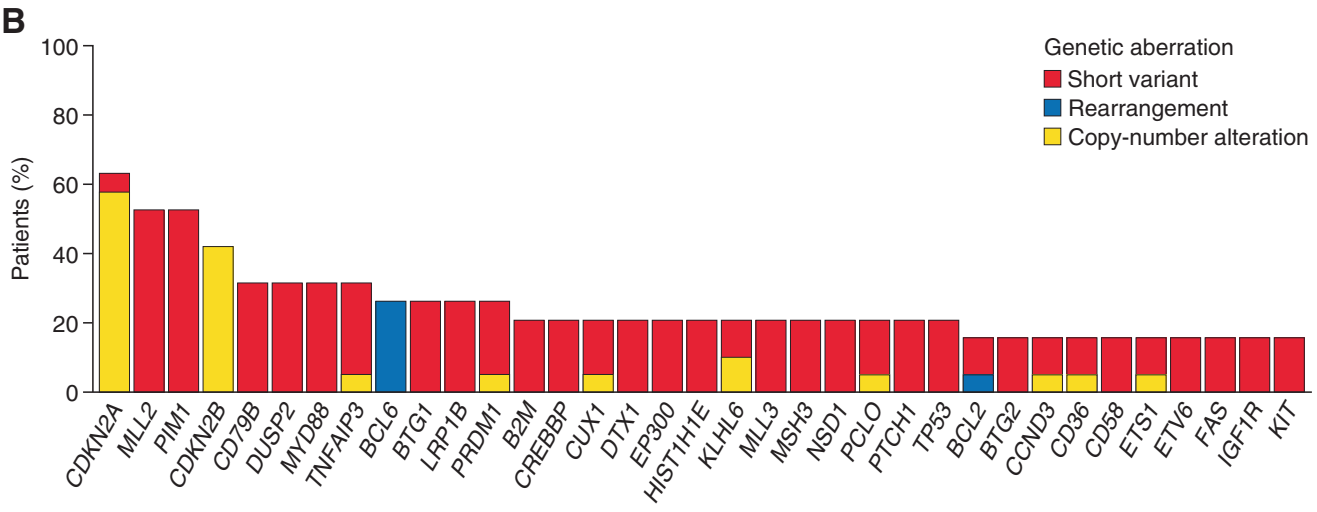

Aberrant gene

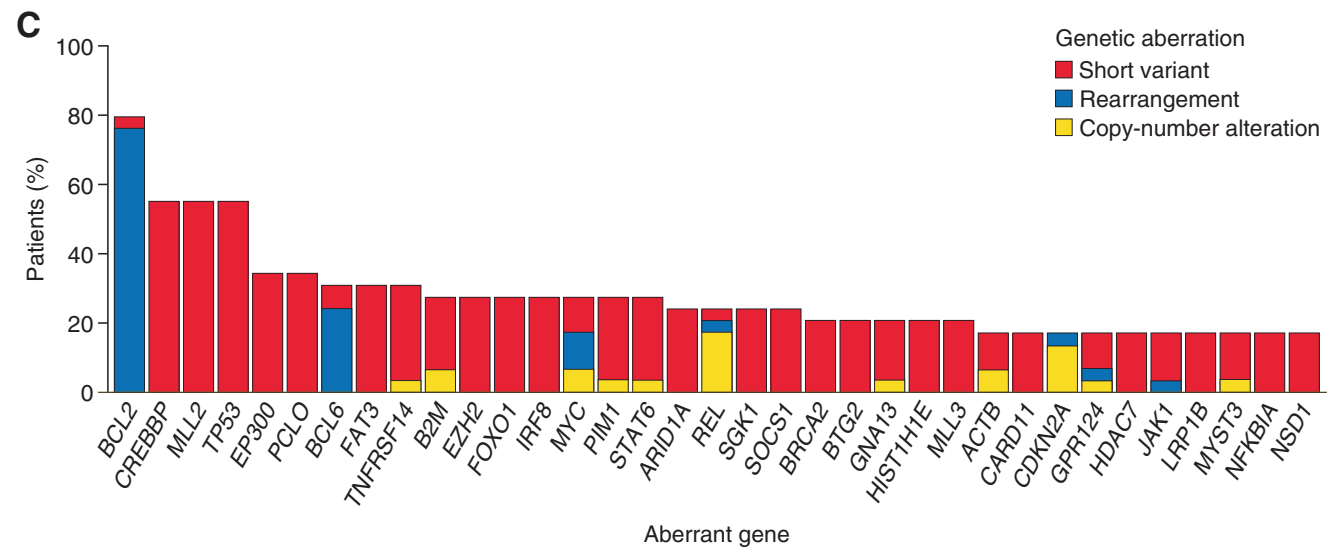

Fig. 3 Most common tumor aberrations detected. a The overall cohort; b ABC DLBCL (overall cohort); and $\mathbf{c}$ GCB DLBCL (overall cohort). ABC activated B-cell like, DLBCL diffuse large B-cell lymphoma, GCB germinal center B-cell like.

11/15 patients with a composite score above the cutoff who had either PR or CR, compared with 0/29 patients with a score below the cutoff who had a response; PFS 5.4 vs. 1.7 months; hazard ratio 2.27 [95\% CI 1.15, 4.49]; $P=$ 0.018 ) (Fig. 4c). The genes in this signature included several with higher prevalence in one molecular subtype (ABC DLBCL vs. GCB DLBCL) than the other (e.g., TNFAIP3, CD58, PRDM1, and CBL in ABC DLBCL and MYST3, CREBBP, SGK1, PARP4, and PDCD11 in GCB DLBCL) (Fig. 4b, Supplementary Fig. 2), as well as several genes with known prognostic significance in DLBCL (TNFAIP3, CD58, CREBBP, and PRDM1).

Overall, the safety analysis set comprised 67 patients; 65 patients $(97.0 \%)$ experienced at least one treatmentemergent AE (TEAE) (Table 3). The most common grade 3 TEAEs were hypertension (32.8\%) and hyperglycemia (22.3\%), and the most common grade 4 event was hyperglycemia $(9.0 \%)$. Most postinfusion hypertension events were grade $\leq 2$ and were transient, manageable, and resolved following treatment; no grade 4 hypertension events were 
A

ORR

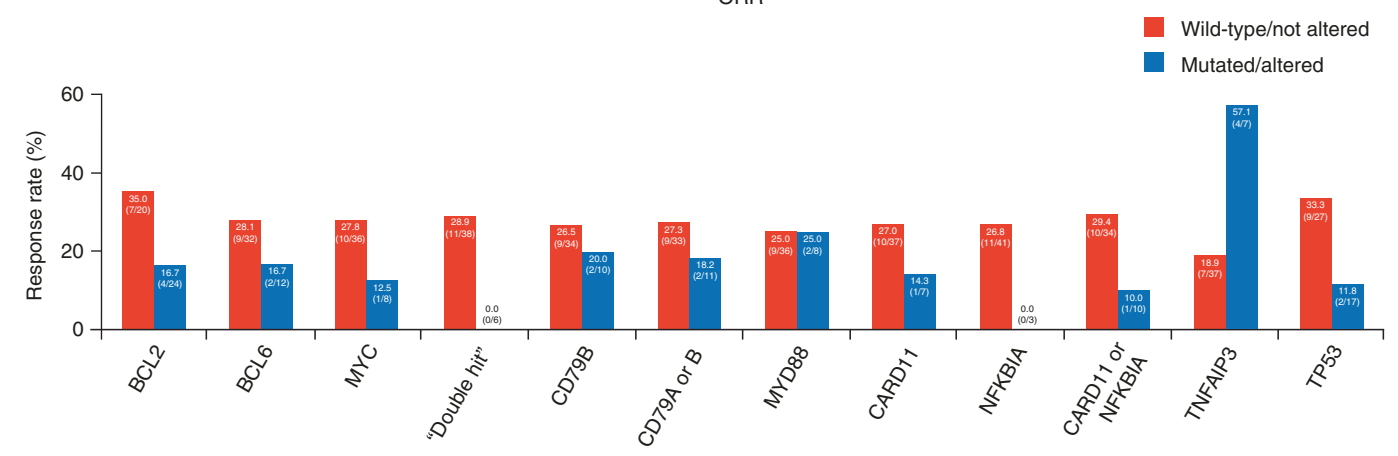

B
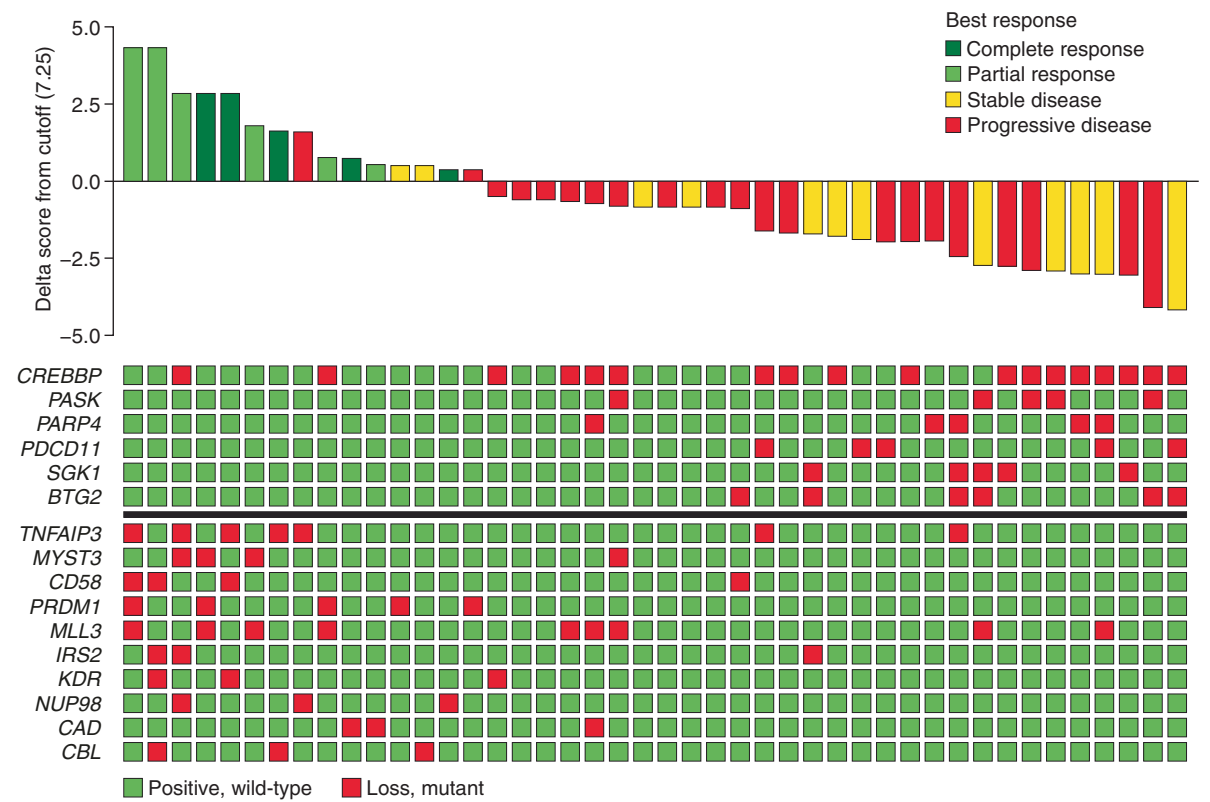

C

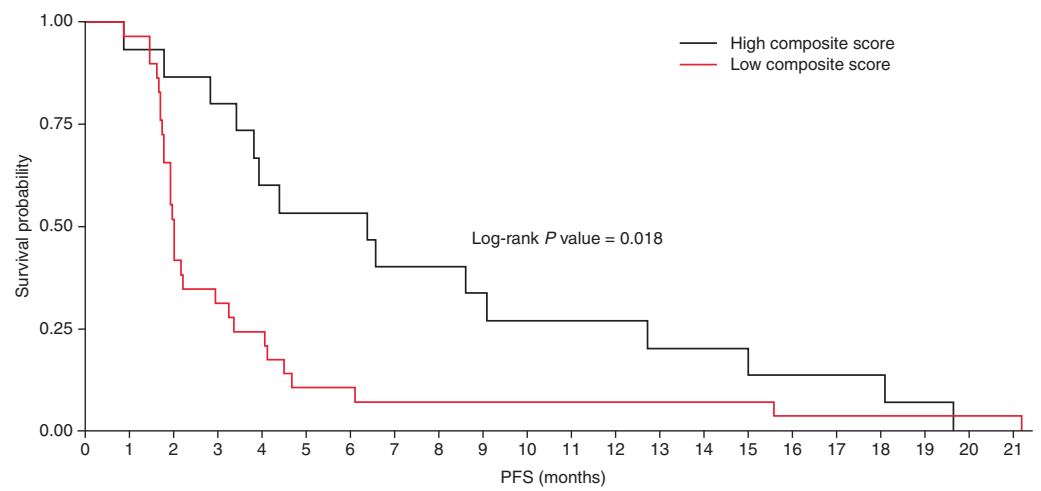

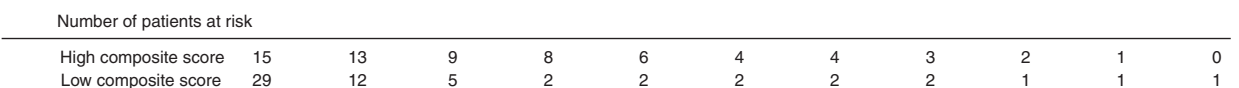

Fig. 4 Correlation of NGS biomarker data with response. a Correlation of mutational status and clinical outcome (all patients with NGS and response data were analyzed; $n=44$ ). b Multigene mutation signature separating responders from nonresponders and their computed composite scores (patients are sorted based on the composite score. Score values are shown as a waterfall of delta scores to the cutoff level. The list of genes with mutations predictive of response is given in the matrix below the waterfall plot. Genes with the presence of a mutation in the responder group [and absence of mutation in the nonresponder groups] contributing to the score are shown on the bottom, while genes with the absence of a mutation in the responder group [or presence of mutation in the nonresponder group] contributing to the score are shown on top) and c PFS by mutation composite score. NGS next-generation sequencing, ORR objective response rate, PFS progression-free survival. 
Table 3 Overview of TEAEs and incidence of TEAEs and drug-related TEAEs occurring in $\geq 10 \%$ of the total population (overall cohort).

\begin{tabular}{|c|c|c|c|c|}
\hline$n(\%)$ & Grade 1 or 2 & Grade 3 or 4 & Grade 5 & $\begin{array}{l}\text { Total } \\
N=67\end{array}$ \\
\hline Any TEAE ${ }^{\mathrm{a}}$ & $7(10.4)$ & $44(65.7)$ & $14(20.9)$ & $65(97.0)$ \\
\hline SAEs & $2(3.0)$ & $28(41.8)$ & $14(20.9)$ & $44(65.7)$ \\
\hline Patients with TEAEs leading to dose reduction & $2(3.0)$ & $7(10.4)$ & 0 & $9(13.4)$ \\
\hline $\begin{array}{l}\text { Patients with TEAEs leading to dose interruption/ } \\
\text { delay }\end{array}$ & $11(16.4)$ & $23(34.3)$ & 0 & $34(50.7)$ \\
\hline $\begin{array}{l}\text { Patients with TEAEs leading to permanent } \\
\text { discontinuation }\end{array}$ & $4(6.0)$ & $13(19.4)$ & 0 & $17(25.4)$ \\
\hline \multicolumn{5}{|c|}{ Incidence of TEAEs occurring in $>10 \%$ of the total population } \\
\hline Hypertension & $5(7.5)$ & $22(32.8)$ & 0 & $27(40.3)$ \\
\hline Diarrhea & $24(35.8)$ & $1(1.5)$ & 0 & $25(37.3)$ \\
\hline Hyperglycemia & $1(1.5)$ & $21(31.3)$ & 0 & $22(32.8)$ \\
\hline Nausea & $20(29.9)$ & $1(1.5)$ & 0 & $21(31.3)$ \\
\hline Fatigue & $18(26.9)$ & 0 & $1(1.5)^{b}$ & $19(28.4)$ \\
\hline Pyrexia & $13(19.4)$ & $1(1.5)$ & 0 & $14(20.9)$ \\
\hline Cough & $11(16.4)$ & $1(1.5)$ & 0 & $12(17.9)$ \\
\hline Vomiting & $11(16.4)$ & $1(1.5)$ & 0 & $12(17.9)$ \\
\hline Constipation & $11(16.4)$ & 0 & 0 & $11(16.4)$ \\
\hline Decreased appetite & $10(14.9)$ & 0 & 0 & $10(14.9)$ \\
\hline Deterioration in general physical health & 0 & $1(1.5)$ & 8 (11.9) & $9(13.4)$ \\
\hline Headache & $9(13.4)$ & 0 & 0 & $9(13.4)$ \\
\hline Neutropenia & $1(1.5)$ & $8(11.9)$ & 0 & $9(13.4)$ \\
\hline Rash & $8(11.9)$ & $1(1.5)$ & 0 & $9(13.4)$ \\
\hline Hypokalemia & $4(6.0)$ & $4(6.0)$ & 0 & $8(11.9)$ \\
\hline Mouth ulceration & $7(10.4)$ & $1(1.5)$ & 0 & $8(11.9)$ \\
\hline Dyspnea & $4(6.0)$ & $3(4.5)$ & 0 & $7(10.4)$ \\
\hline Peripheral edema & $7(10.4)$ & 0 & 0 & $7(10.4)$ \\
\hline \multicolumn{5}{|c|}{ Incidence of drug-related TEAEs occurring in $>10 \%$ of the total population } \\
\hline Hypertension & $4(6.0)$ & $19(28.4)$ & 0 & $23(34.3)$ \\
\hline Hyperglycemia & $1(1.5)$ & $20(29.9)$ & 0 & $21(31.3)$ \\
\hline Fatigue & $12(17.9)$ & 0 & 0 & $12(17.9)$ \\
\hline Nausea & $12(17.9)$ & 0 & 0 & $12(17.9)$ \\
\hline Diarrhea & $10(14.9)$ & $1(1.5)$ & 0 & $11(16.4)$ \\
\hline Vomiting & $7(10.4)$ & $1(1.5)$ & 0 & $8(11.9)$ \\
\hline Mouth ulceration & $6(9.0)$ & $1(1.5)$ & 0 & $7(10.4)$ \\
\hline Neutropenia & $1(1.5)$ & $6(9.0)$ & 0 & $7(10.4)$ \\
\hline
\end{tabular}

SAE serious adverse event, TEAE treatment-emergent adverse event.

${ }^{\text {a }}$ Specified event starting or worsening between start of treatment and 30 days after the end of treatment.

${ }^{\mathrm{b}}$ This patient received treatment with copanlisib for 14 days and was withdrawn from the study due to disease progression. The patient experienced increasing fatigue that was considered an SAE due to hospitalization occurring 15 days after the last dose of copanlisib, with a reported grade 5 (fatal) outcome occurring 2 days later, considered as a symptom of disease progression. The event was considered unrelated to treatment with copanlisib or protocol-required procedures, with the primary cause of death reported as underlying disease.

reported. Results were similar in patients with and without a medical history of hypertension. The prevalence of postinfusion hyperglycemia events was highest in cycle 1 , after which new hyperglycemia events occurred at a lower rate $(80.6 \%, 46.3 \%$, and $16.4 \%$ on day 1 of cycles 1,2 , and 3 , respectively; data not shown for remaining cycles).
Serious AEs (SAEs) occurred in 44 patients (65.7\%). Twenty-two patients $(32.8 \%)$ experienced grade 3 SAEs, six patients $(9.0 \%)$ experienced grade 4 SAEs, and all 14 grade 5 events $(20.9 \%)$ were considered SAEs. Fifty-five patients $(82.1 \%)$ experienced drug-related TEAEs, most commonly hypertension (34.3\%), hyperglycemia (31.3\%), 
and fatigue and nausea (17.9\% each) (Table 3$)$. Three patients $(4.5 \%)$ experienced pneumonitis (two patients [3.0\%] with grade 3 and one patient [1.5\%] with grade 4), all considered drug related. Five patients $(7.5 \%)$ experienced a drug-related infection or infestation of grade $\geq 3$. One patient experienced drug-related grade 2 Clostridium colitis. The most common laboratory abnormalities were hyperglycemia $(63 / 67,94.0 \%)$, anemia $(61 / 65,93.8 \%)$, lymphocytopenia $(54 / 65,83.1 \%)$, thrombocytopenia (38/ $65,58.5 \%)$, leukocytopenia $(37 / 65,56.9 \%)$, and neutropenia $(29 / 65,44.6 \%)$.

Hyperglycemia TEAEs were present at a higher incidence in the 11 patients with a confirmed diagnosis of diabetes compared with patients without a history of diabetes, with grade 3 hyperglycemia experienced by $63.6 \%$ (7/11) and 21.4\% (12/56) of patients, respectively. Grade 4 hyperglycemia was experienced by $27.3 \%$ (3/11) and 5.4\% (3/56) of patients, respectively. Mean change in glycated hemoglobin from baseline was slightly higher in diabetic patients $(1.50 \pm 0.85 \% ; n=8)$ than in nondiabetic patients $(0.49 \pm 0.98 \% ; n=41)$. Most postinfusion hyperglycemia events after cycle 1 , day 1 were grade $<2$, with no grade 4 events reported in any patients.

TEAEs leading to permanent discontinuation occurred in $25.4 \%$ of patients and were considered drug related in $11.9 \%$ (Table 3), including one patient (1.5\%) each with asthenia, thrombocytopenia, hyperglycemia, hyponatremia, hypophosphatemia, pneumonitis, rash, and Stevens-Johnson syndrome. No patient discontinued treatment due to hypertension. One patient each discontinued therapy due to hyperglycemia and pneumonitis. Fourteen grade 5 TEAEs (20.9\%) were reported. None of these were considered drug related. Patient deaths were mostly due to disease progression (43 patients, $64.2 \%$ ).

Dose interruptions or delays, $91 \%$ of which were due to AEs, were experienced by 38 patients $(56.7 \%)$. Of these dose interruptions or delays, $94 \%$ lasted $\leq 1$ week (median 0.79 weeks [range $0-2.0]$ ) and nine patients $(13.4 \%$ ) had a dose reduction to $45 \mathrm{mg}$, mostly due to AEs. The most common reason for treatment discontinuation was radiologic or clinical progressive disease $(49 / 67,73.1 \%)$.

\section{Discussion}

For patients with relapsed/refractory DLBCL, treatment options are still very limited, presenting an urgent need for novel therapeutic approaches. In this phase II study, monotherapy treatment with copanlisib demonstrated an ORR of $19.4 \%$, a response comparable with response rates reported with other nonchemotherapy single agents in unselected DLBCL, including the immunomodulatory agent lenalidomide (27.5\%) [25] and the Bruton's tyrosine kinase inhibitor ibrutinib (25\%) [26].
These clinical data reflect previous preclinical in vitro and in vivo studies that have suggested stronger activity of PI3K- $\alpha /-\delta$ inhibition in ABC DLBCL compared with GCB DLBCL models [13-15, 17]. The results are generally consistent with these findings, with copanlisib demonstrating a numerically higher response rate in ABC DLBCL compared with GCB DLBCL patients. In the overall cohort, patients with ABC DLBCL demonstrated an ORR of $31.6 \%$, compared with $13.3 \%$ in patients with GCB DLBCL, though the difference was not statistically significant $(P=0.1413)$. These data suggest that despite the worse prognosis expected for this group, ABC DLBCL may respond preferentially to pharmacologic inhibition of oncogenic PI3K signaling. The results of our study are comparable to those reported for ibrutinib (response rate of $37 \%$ for ABC DLBCL and 5\% for GCB DLBCL) and lenalidomide (response rate of $46 \%$ for $A B C$ DLBCL and $21.4 \%$ for GCB DLBCL) $[25,26]$.

Copanlisib elicited similar responses in patients with and without $C D 79 B$ mutations, indicating modest antilymphoma activity regardless of the presence of chronic BCR signaling. These results echo those from a trial investigating the efficacy of ibrutinib monotherapy in relapsed/refractory DLBCL patients, in which patients with mutated and wildtype $C D 79 B$ responded similarly to treatment [26]. Further exploratory mutational analyses highlighted that several mutations modifying oncogenic nuclear factor- $\mathrm{KB}$ pathway signaling may have an impact on the efficacy of copanlisib. While we determined that MYD88 mutations do not influence response, the mutational status of TNFAIP3, NFKBIA, and CARDI1 might be associated with sensitivity to copanlisib. In addition, genetic aberrations in other genes seem to influence response to copanlisib. Patients with $B C L 2$ mutations had a numerically lower response rate, and no patient with alterations in $M Y C$ plus either $B C L 2$ or $B C L 6$ achieved an objective response.

Using an unbiased approach, we were able to identify a 16gene mutation signature separating copanlisib responders from nonresponders. Though exploratory in nature, this signature might be utilized in the future to select patients who are treated with copanlisib. Though not all genes in the signature have a known prognostic role in DLBCL, it cannot be ruled out that the signature may be separating prognostic groups, where patients with good prognosis may have a higher likelihood of responding to therapy than those with poor prognosis, which could result in a better response rate and longer PFS. However, whether these findings reflect prognostic influence conferred by these mutations or a predictive ability for copanlisib activity could not be determined in the present study due to the small groups and lack of a control arm. These results provide the basis for future validation and analyses.

Recently, several large genetic studies identified several novel molecular DLBCL subtypes [27-29]. One of these 
studies identified five DLBCL subsets based on recurrent mutations, somatic copy-number alterations, structural variants, and defined coordinate signatures [27]. Another study described four prominent genetic subtypes in DLBCL, termed MCD (based on the co-occurrence of MYD88L265P and $C D 79 B$ mutations), BN2 (based on BCL6 fusions and NOTCH2 mutations), N1 (based on NOTCH1 mutations), and EZB (based on EZH2 mutations and BCL2 translocations), using exome and transcriptome sequencing, array-based DNA copy-number analysis, and targeted amplicon resequencing [29]. It will be interesting to investigate in the future if these specific molecular subsets of DLBCL will respond preferentially to copanlisib or other PI3K inhibitors.

Overall, copanlisib demonstrated a safety profile that was recognizable and manageable. TEAEs most commonly reported were hypertension, diarrhea, and hyperglycemia, consistent with toxicities observed with copanlisib in previous trials [19, 20, 30]. Hyperglycemia events were generally grade $<4$. Hyperglycemia is likely to be an on-target effect of the inhibition of the PI3K- $\alpha$ isoform, related to downstream signaling of insulin receptors, leading to transient, reduced tissue utilization of glucose, and/or insulin resistance [31]. Hypertension events were never grade 4 in severity and did not lead to discontinuation in any of the affected patients. The molecular mechanisms of copanlisib leading to hypertension are currently unclear. However, the majority of hypertension events observed in patients treated with copanlisib were transient, which may be related to acute vasoconstriction resulting from the intravenous infusion of copanlisib [32]. Overall, hyperglycemia and hypertension were transient and considered manageable, with almost all patients reporting values approaching baseline prior to subsequent infusions, consistent with previous pharmacodynamic studies [30].

In conclusion, single-agent copanlisib demonstrated modest antilymphoma activity in patients with relapsed/ refractory DLBCL with a numerically higher response rate in patients with $\mathrm{ABC}$ DLBCL vs. patients with GCB DLBCL, with similar activity in patients with and without $C D 79 B$ mutations. A 16-gene mutation signature associated with improved outcomes was identified as a possible approach (with further validation) for selecting DLBCL patients for treatment with copanlisib. Copanlisib showed an acceptable safety profile, with the most common toxicities of hypertension and hyperglycemia being transient and manageable. To our knowledge, this is the largest phase II study on the use of a PI3K inhibitor in patients with DLBCL; therefore, further exploration of copanlisib in combination with other targeted therapies (e.g., a Bruton's tyrosine kinase inhibitor [14] or a BCL2 antagonist [15]) or in molecularly defined subgroups of DLBCL is warranted. In addition, a phase II study evaluating copanlisib in combination with rituximab-CHOP chemotherapy in previously untreated patients with DLBCL will be initiated in 2020 (EudraCT 2018-003560-31).

\section{Data availability}

Availability of the data underlying this publication will be determined according to Bayer's commitment to the EFPIA/ PhRMA "Principles for responsible clinical trial data sharing". This pertains to scope, time point, and process of data access.

As such, Bayer commits to sharing upon request from qualified scientific and medical researchers patient-level clinical trial data, study-level clinical trial data, and protocols from clinical trials in patients for medicines and indications approved in the United States (US) and European Union (EU) as necessary for conducting legitimate research. This applies to data on new medicines and indications that have been approved by the EU and US regulatory agencies on or after January 1, 2014.

Interested researchers can use www.clinicalstudydata request.com to request access to anonymized patient-level data and supporting documents from clinical studies to conduct further research that can help advance medical science or improve patient care. Information on the Bayer criteria for listing studies and other relevant information is provided in the study sponsors section of the portal.

Data access will be granted to anonymized patient-level data, protocols, and clinical study reports after approval by an independent scientific review panel. Bayer is not involved in the decisions made by the independent review panel. Bayer will take all necessary measures to ensure that patient privacy is safeguarded.

Acknowledgements This study was supported by research funding from Bayer AG. Bayer had a role in study design, data analysis, data interpretation, writing of the report, and decision to submit the paper for publication. Bayer had no role in data collection. The corresponding author (GL) had full access to all data and had final responsibility for the decision to submit for publication. Jack Adams, MSc, at Complete HealthVizion (Manchester, UK) provided medical writing assistance with this paper, based on detailed discussion and feedback from all the authors; this assistance was funded by Bayer AG. We thank Michael Pawlack and Oliver Wirtz for statistical programming in this study. Open access funding provided by Projekt DEAL.

Author contributions Research design: GL, IG, SL, FH, GB, CP, VB, and BHC; data collection and assembly: EH, CH, STL, DSH, KA, GC, GB, BHC, and GS; data analysis: GL, EH, GV, KA, JH, WS, SL, FH, $\mathrm{PP}, \mathrm{GB}, \mathrm{CP}, \mathrm{VB}$, and $\mathrm{BHC}$; writing of the paper and approving for submission: all authors.

\section{Compliance with ethical standards}

Conflict of interest GL has received honoraria from AstraZeneca, Bayer, Bristol-Myers Squibb, Celgene, Gilead, Hexal, Janssen, MorphoSys, NanoString, and Roche, has participated in a consulting or advisory role for Amgen, AstraZeneca, Bayer, Bristol-Myers Squibb, 
Celgene, Gilead, Hexal, Janssen, MorphoSys, NanoString, Novartis, and Roche, has been on a speaker's bureau for Bayer, Celgene, Gilead, Janssen, and Roche, has received research funding from AstraZeneca, Bayer, Celgene, Gilead, Janssen, and Roche, and has had travel, accommodation, and expenses reimbursed by Bayer, Celgene, Gilead, Hexal, Janssen, and Roche. EH has participated in a consulting or advisory role for Bristol-Myers Squibb, Celgene, and Janssen, has been on a speaker's bureau for Bristol-Myers Squibb, Janssen, and Roche, has received research funding from Bristol-Myers Squibb, Celgene, Merck Serono, and Merck Sharp \& Dohme, and has had travel, accommodation, and expenses reimbursed by Bristol-Myers Squibb, Roche, and Takeda. $\mathrm{CH}$ has received honoraria from Celgene, Gilead, Janssen, Novartis, Roche, and Takeda, has participated in a consulting or advisory role for Celgene, Gilead, Janssen, Roche, and Takeda, and has had travel, accommodation, and expenses reimbursed by Celgene and Roche. KA has received honoraria from Takeda, has participated in a consulting or advisory role for Celgene, Gilead, Roche, and Takeda, has received research funding from Cell Medica, Gilead, Infinity, Merck, and Pharmacyclics, has had travel, accommodation, and expenses reimbursed by Celgene, Gilead, Roche, and Takeda, and is supported by the UCLH/ UCL Biomedical Research Centre. GC has received research funding from Bayer HealthCare Pharmaceuticals, Bristol-Myers Squibb, Hutchison, Incyte, Merck Serono, and Novartis. WS is employed by Bayer HealthCare Pharmaceuticals. IG was employed by Bayer HealthCare Pharmaceuticals. SL is employed by, and has stock or other ownership at, Bayer AG. FH is employed by Bayer AG and has stock or other ownership at Bayer AG, Evotec AG, Gilead, Hologen AG, Medigene AG, and 4SC AG. PP is employed by, and has stock or other ownership at, Bayer AG. GB is employed by Bayer AG. CP is employed by, has stock at, and has co-authored patents at Bayer HealthCare Pharmaceuticals. VB is employed by Bayer HealthCare Pharmaceuticals. BHC is employed by Bayer HealthCare Pharmaceuticals. GS has received honoraria from Acerta, Amgen, Bristol-Myers Squibb, Celgene, Gilead, Janssen, Merck, MorphoSys, Novartis, Pfizer, Roche, and Servier, has participated in a consulting or advisory role for Amgen, Celgene, Gilead, Merck, Novartis, Pfizer, Roche, and Servier, has received research funding from Acerta, Amgen, Bristol-Myers Squibb, Celgene, Gilead, Janssen, Merck, MorphoSys, Novartis, Pfizer, Roche, Sanofi, Servier, and Takeda, and has had travel, accommodation, and expenses reimbursed by Roche and Sanofi. GV, STL, DSH, and JH declare no competing financial interests.

Publisher's note Springer Nature remains neutral with regard to jurisdictional claims in published maps and institutional affiliations.

Open Access This article is licensed under a Creative Commons Attribution 4.0 International License, which permits use, sharing, adaptation, distribution and reproduction in any medium or format, as long as you give appropriate credit to the original author(s) and the source, provide a link to the Creative Commons license, and indicate if changes were made. The images or other third party material in this article are included in the article's Creative Commons license, unless indicated otherwise in a credit line to the material. If material is not included in the article's Creative Commons license and your intended use is not permitted by statutory regulation or exceeds the permitted use, you will need to obtain permission directly from the copyright holder. To view a copy of this license, visit http://creativecommons. org/licenses/by/4.0/.

\section{References}

1. Nogai H, Dörken B, Lenz G. Pathogenesis of non-Hodgkin's lymphoma. J Clin Oncol. 2011;29:1803-11.
2. Sehn LH, Gascoyne RD. Diffuse large B-cell lymphoma: optimizing outcome in the context of clinical and biologic heterogeneity. Blood. 2015;125:22-32.

3. Coiffier B, Lepage E, Brière J, Herbrecht R, Tilly H, Bouabdallah $\mathrm{R}$, et al. CHOP chemotherapy plus rituximab compared with CHOP alone in elderly patients with diffuse large-B-cell lymphoma. N Engl J Med. 2002;346:235-42.

4. Pfreundschuh M, Trümper L, Österborg A, Pettengell R, Trneny $\mathrm{M}$, Imrie K, et al. CHOP-like chemotherapy plus rituximab versus CHOP-like chemotherapy alone in young patients with goodprognosis diffuse large-B-cell lymphoma: a randomised controlled trial by the MabThera International Trial (MInT) Group. Lancet Oncol. 2006;7:379-91.

5. Friedberg JW. Relapsed/refractory diffuse large B-cell lymphoma. Hematol Am Soc Hematol Educ Progr. 2011;2011:498-505.

6. Crump M, Neelapu SS, Farooq U, Van Den Neste E, Kuruvilla J, Westin $\mathrm{J}$, et al. Outcomes in refractory diffuse large B-cell lymphoma: results from the international SCHOLAR-1 study. Blood. 2017;130:1800-8.

7. Gisselbrecht C, Glass B, Mounier N, Singh Gill D, Linch DC, Trneny M, et al. Salvage regimens with autologous transplantation for relapsed large B-cell lymphoma in the rituximab era. J Clin Oncol. 2010;28:4184-90.

8. Kondo E. Autologous hematopoietic stem cell transplantation for diffuse large B-cell lymphoma. J Clin Exp Hematop. 2016;56:100-8.

9. Alizadeh AA, Eisen MB, Davis RE, Ma C, Lossos IS, Rosenwald A, et al. Distinct types of diffuse large B-cell lymphoma identified by gene expression profiling. Nature. 2000;403:503-11.

10. Lenz G, Staudt LM. Aggressive lymphomas. N Engl J Med. 2010;362:1417-29.

11. Lenz G, Wright G, Dave SS, Xiao W, Powell J, Zhao H, et al. Stromal gene signatures in large B-cell lymphomas. N Engl J Med. 2008;359:2313-23.

12. Davis RE, Ngo VN, Lenz G, Tolar P, Young RM, Romesser PB, et al. Chronic active B-cell-receptor signalling in diffuse large Bcell lymphoma. Nature. 2010;463:88-92.

13. Erdmann T, Klener P, Lynch JT, Grau M, Vočková P, Molinsky J, et al. Sensitivity to PI3K and AKT inhibitors is mediated by divergent molecular mechanisms in subtypes of DLBCL. Blood. 2017;130:310-22.

14. Paul J, Soujon M, Wengner AM, Zitzmann-Kolbe S, Sturz A, Haike K, et al. Simultaneous inhibition of PI3K $\delta$ and PI3K $\alpha$ induces $\mathrm{ABC}$-DLBCL regression by blocking BCR-dependent and -independent activation of NF- $\mathrm{KB}$ and AKT. Cancer Cell. 2017;31:64-78.

15. Bojarczuk K, Wienand K, Ryan JA, Chen L, Villalobos-Ortiz M, Mandato E, et al. Targeted inhibition of $\mathrm{PI} 3 \mathrm{~K} \alpha / \delta$ is synergistic with BCL-2 blockade in genetically defined subtypes of DLBCL. Blood. 2019;133:70-80.

16. Kahl B, Byrd JC, Flinn IW, Wagner-Johnston N, Spurgeon S, Benson DM Jr, et al. Clinical safety and activity in a Phase 1 study of CAL-101, an isoform-selective inhibitor of phosphatidylinositol 3-kinase $\mathrm{P} 110 \delta$, in patients with relapsed or refractory nonHodgkin lymphoma. Blood. 2010;116:1777.

17. Pongas GN, Annunziata CM, Staudt LM. PI3K $\delta$ inhibition causes feedback activation of $\mathrm{PI} 3 \mathrm{~K} \alpha$ in the ABC subtype of diffuse large B-cell lymphoma. Oncotarget. 2017;8:81794-802.

18. Haike K, Stasik E, Soujon M, Wengner AM, Petrova E, Genvresse $\mathrm{I}$, et al. Molecular mechanisms supporting inhibition of PI3K isoforms by copanlisib in blocking B-cell signaling and tumor cell growth in diffuse large B-cell lymphoma. Poster 48 presented at 1st American Society of Hematology Meeting on Lymphoma Biology, Colorado Springs, Colorado, USA, August 10-13, 2014. 
19. Patnaik A, Appleman LJ, Tolcher AW, Papadopoulos KP, Beeram M, Rasco DW, et al. First-in-human phase I study of copanlisib (BAY 80-6946), an intravenous pan-class I phosphatidylinositol 3-kinase inhibitor, in patients with advanced solid tumors and non-Hodgkin's lymphomas. Ann Oncol. 2016;27:1928-40.

20. Dreyling M, Morschhauser F, Bouabdallah K, Bron D, Cunningham D, Assouline SE, et al. Phase II study of copanlisib, a $\mathrm{PI} 3 \mathrm{~K}$ inhibitor, in relapsed or refractory, indolent or aggressive lymphoma. Ann Oncol. 2017;28:2169-78.

21. Dreyling M, Santoro A, Mollica L, Leppä S, Follows GA, Lenz G, et al. Phosphatidylinositol 3-kinase inhibition by copanlisib in relapsed or refractory indolent lymphoma. J Clin Oncol. 2017;35:3898-905.

22. Campo E, Swerdlow SH, Harris NL, Pileri S, Stein H, Jaffe ES. The 2008 WHO classification of lymphoid neoplasms and beyond: evolving concepts and practical applications. Blood. 2011;117:5019-32.

23. Cheson BD, Fisher RI, Barrington SF, Cavalli F, Schwartz LH, Zucca E, et al. Recommendations for initial evaluation, staging, and response assessment of Hodgkin and non-Hodgkin lymphoma: the Lugano classification. J Clin Oncol. 2014;32:3059-67.

24. Perrière G, Lobry JR, Thioulouse J. Correspondence discriminant analysis: a multivariate method for comparing classes of protein and nucleic acid sequences. Comput Appl Biosci. 1996;12: 519-24.

25. Czuczman MS, Trněný M, Davies A, Rule S, Linton KM, Wagner-Johnston N, et al. A phase $2 / 3$ multicenter, randomized, open-label study to compare the efficacy and safety of lenalidomide versus investigator's choice in patients with relapsed or refractory diffuse large B-cell lymphoma. Clin Cancer Res. 2017;23:4127-37.

26. Wilson WH, Young RM, Schmitz R, Yang Y, Pittaluga S, Wright $\mathrm{G}$, et al. Targeting B cell receptor signaling with ibrutinib in diffuse large B cell lymphoma. Nat Med. 2015;21:922-6.

27. Chapuy B, Stewart C, Dunford AJ, Kim J, Kamburov A, Redd RA, et al. Molecular subtypes of diffuse large B cell lymphoma are associated with distinct pathogenic mechanisms and outcomes. Nat Med. 2018;24:679-90.

28. Reddy A, Zhang J, Davis NS, Moffitt AB, Love CL, Waldrop A, et al. Genetic and functional drivers of diffuse large B cell lymphoma. Cell. 2017;171:481-94.e15.

29. Schmitz R, Wright GW, Huang DW, Johnson CA, Phelan JD, Wang JQ, et al. Genetics and pathogenesis of diffuse large B-cell lymphoma. N Engl J Med. 2018;378:1396-407.

30. Morschhauser F, Machiels JP, Salles G, Rottey S, Rule SAJ, Cunningham $\mathrm{D}$, et al. On-target pharmacodynamic activity of the PI3K inhibitor copanlisib in paired biopsies from patients with malignant lymphoma and advanced solid tumors. Mol Cancer Ther. 2019. https://doi.org/10.1158/1535-7163.Mct-19-0466.

31. Shepherd PR, Withers DJ, Siddle K. Phosphoinositide 3-kinase: the key switch mechanism in insulin signalling. Biochem $\mathrm{J}$. 1998;333:471-90.

32. Northcott CA, Poy MN, Najjar SM, Watts SW. Phosphoinositide 3-kinase mediates enhanced spontaneous and agonist-induced contraction in aorta of deoxycorticosterone acetate-salt hypertensive rats. Circ Res. 2002;91:360-9. 